\title{
Pequena produção orgânica no Brasil: a tecnopolítica dominante da agroecologia
}

\author{
Orlando Gomes da Mestre em Engenharia de Produção. Universidade Federal de Campina Grande \\ Silva (UFCG)-Brasil.02@riseup.net
}

\section{RESUMO}

A produção de orgânicos no Brasil está associada, majoritariamente, a pequenos produtores e à agroecologia. Este ensaio, a partir da contextualização do amplo espectro da pequena agricultura orgânica, tem por objetivo problematizar uma interpretação da estrutura de significados dados à agroecologia como a tecnologia de produção e organizacional praticamente oficial para os pequenos produtores de orgânicos no Brasil. Para tanto, utiliza-se metodologicamente uma composição teórica em torno do conceito de tecnologia como realização e apresenta-se o que se vê como a estrutura tecnológica dominante nas definições da política pública brasileira de produção orgânica. Conclui-se que, frente às dificuldades com a assistência técnica, enviezada pela agroecologia, no que tange principalmente a circuitos de comercialização, há uma necessidade de se desenvolver pesquisa sobre outros modelos tecnológicos de produção e gestão para as diferentes pequenas agriculturas orgânicas.

Palavras-chave: Produtos orgânicos. Tecnologia organizacional. Gestão de políticas públicas.

\section{Small organic production in Brazil: the dominant technopolitics of agroecology}

\begin{abstract}
Organic production in Brazil is associated, in large part, with small producers and agroecology. This essay, based on the contextualization of the broad spectrum of small organic agriculture, aims to discuss the structure of meanings given to agroecology as the practically official production and organizational technology for small organic producers in Brazil. Thus, a theoretical composition around the concept of technology as an accomplishment is adopted methodologically and it is presented as is seen as the dominant technological structure in the definitions of Brazilian public policy for organic production. It is concluded that, faced with the difficulties in technical assistance concerning mainly marketing circuits, there is a need to develop research on other technological models of production and management for the different small organic farms.
\end{abstract}

Keywords: Organic products. Organizational technology. Public policy administration. 


\section{INTRODUÇÃO}

Os produtos orgânicos vêm chamando a atenção mundial nas últimas décadas, atraindo consumidores conscientes dos problemas com a produção industrial de alimentos, atendendo a um público que pode se dar ao luxo de consumo ainda sofisticado e, ao mesmo tempo, constituindo interesses e práticas que, no âmbito nacional, recebem uma delimitação abrangente com Lei no 10.831/2003 (BRASIL, 2003), sua posterior regulamentação com o Decreto no 6.323/2007 (BRASIL, 2007) e o estabelecimento da Política Nacional de Agroecologia e Produção Orgânica - PNAPO, cujo objetivo é "integrar, articular e adequar políticas, programas e ações indutoras da transição agroecológica e da produção orgânica e de base agroecológica, contribuindo para o desenvolvimento sustentável e a qualidade de vida da população, por meio do uso sustentável dos recursos naturais e da oferta e consumo de alimentos saudáveis" (BRASIL, 2012).

Nesse contexto, há a noção de que a grande maioria dos produtores orgânicos, 83 por cento de acordo com último censo agropecuário em 2006 (INSTITUTO DE PROMOÇÃO DO DESENVOLVIMENTO, 2010), concentra-se no que no Brasil é considerada a agricultura familiar, ou seja, aqueles que geralmente também são tidos como pequenos produtores, ainda que essa associação entre pequeno e familiar possa ser questionada com base nas próprias discussões do que vem a ser agricultura familiar no Brasil (GUANZIROLI; BUAINAIN; DI SABBATO, 2012), que apresenta distintas subclassificações, incluindo produções que não se caracterizam, seja pelo tamanho da área, pela renda obtida ou outros fatores, propriamente como pequenas.

Em um processo de décadas de ações e negociações (MEDAETS; FONSECA, 2005), a chamada produção de base agroecológica estabeleceu-se, no texto da legislação, como o modelo adequado de transição das ditas produções convencionais para a produção de orgânicos, notadamente com foco em pequenas produções e de acordo com compreensões do que seja a agricultura familiar no Brasil, praticamente colocando a agroecologia no posto oficial de tecnologia de produção e gestão da pequena agricultura orgânica.

Porém, as especificações da PNAPO, no que concerne ao que se observa como pequena produção de orgânicos, podem ser problemáticas, porque não abordam questões específicas da administração da produção e comercialização dos pequenos produtores que não se encaixam nas configurações da agroecologia, uma vez que as possibilidades tecnológicas da agricultura orgânica podem vir de diferentes bases (DAROLT, 2010), especificações de certificação e características de comercialização que se mostram bastante problemáticas para os pequenos produtores, especialmente nas relações com supermercados e distribuidores (BLANC; KLEDAL, 2012).

Um olhar para a questão com enfoque nas relações entre ciência, tecnologia e sociedade, entendendo as tecnologias como construções, como realizações a partir das relações cotidianas (LATOUR; HERMANT, 2010), mostra que escolhas tecnológicas não são apenas definições técnicas racionais e que as trajetórias de estabelecimento das tecnologias permitem o questionamento das abordagens dominantes e a busca por alternativas. Dessa forma, objetiva-se com este ensaio mostrar uma possível interpretação da estrutura de significados dados à agroecologia que a colocam como uma espécie de tecnologia de gestão oficial para os pequenos produtores de orgânicos. Sendo que o termo "estrutura de significados" refere-se a uma visão construcionista da tecnologia, que afirma haver um conjunto de compreensões, uma estrutura, que dá significado às ações dos diferentes atores envolvidos com as tecnologias (PINCH; BIJKER, 1987).

Para o alcance do objetivo proposto organiza-se o trabalho em mais três seções além desta introdução. Primeiro, na seção a seguir, trata-se da pequena produção de orgânicos, destacando seus aspectos legais com 
indicações de diferentes possibilidades tecnológicas. Na seção textual posterior mostra-se a compreensão de tecnologia como realização, que enfatiza as retóricas conformando a tecnologia. Por fim, delineia-se as considerações da estrutura tecnológica da agroecologia, antes das conclusões apontando indicações para o andamento de pesquisas correlatas.

\section{O COMPLEXO ESPECTRO DOS PEQUENOS ORGÂNICOS}

As produções de orgânicos têm suas bases em diferentes práticas desenvolvidas ao redor do mundo durante o século XX, desde a agricultura biodinâmica na Alemanha e na Áustria dos anos vinte ao que se chamou de agricultura orgânica nos anos trinta e quarenta na Grã Bretanha e nos Estados Unidos, passando pela agricultura natural dos anos trinta no Japão, que mais tarde veio a orientar a permacultura na Austrália dos anos setenta e oitenta (DAROLT, 2010).

$\mathrm{Na}$ sequência surge o termo agricultura alternativa agrupando essas diferentes práticas em contraponto à chamada agricultura convencional, que se consolidou a partir dos anos sessenta como estratégia agrícola mundial baseada em pacotes tecnológicos envolvendo sementes de alto desempenho e outros recursos biológicos, insumos químicos e máquinas agrícolas. Da mesma maneira os termos agricultura ecológica e agricultura sustentável, assim como o termo agricultura alternativa, servem para agrupar diferentes agriculturas ligadas a noções de ecologia ou de desenvolvimento sustentável (DAROLT, 2010).

Todavia, no Brasil contemporâneo, o que se chama de produção orgânica recebe uma delimitação abrangente com a Lei $n^{\circ}$ 10.831/2003 (BRASIL, 2003), sua posterior regulamentação com o Decreto no 6.323/2007 (BRASIL, 2007) e o estabelecimento da Política Nacional de Agroecologia e Produção Orgânica PNAPO, que tem por objetivo "integrar, articular e adequar políticas, programas e ações indutoras da transição agroecológica e da produção orgânica e de base agroecológica" (BRASIL, 2012).

A legislação brasileira especifica que o "conceito de sistema orgânico de produção agropecuária e industrial abrange os denominados: ecológico, biodinâmico, natural, regenerativo, biológico, agroecológicos, permacultura e outros que atendam os princípios estabelecidos por esta Lei" (BRASIL, 2003). Dessa forma, pode-se entender a agroecologia como uma possível orientação tecnológica para a produção de orgânicos, mas não como um termo intercambiável para tal produção. E essa distinção é nítida uma vez que a legislação separa as duas coisas e o produto agroecológico não necessita se submeter aos mecanismos de controle dispostos pela Lei nº 10.831/2003 e sua regulamentação (BRASIL, 2012).

Já para a produção do que pode ser denominado como orgânico os mecanismos de controle são mandatórios, podendo ser de dois tipos: a) controle social na venda direta sem certificação, que permite exclusivamente para agricultores familiares "vinculados a uma organização com controle social cadastrada no Ministério da Agricultura, Pecuária e Abastecimento ou em outro órgão fiscalizador federal, estadual ou distrital conveniado" (BRASIL, 2007), identificar seu produto como orgânico apenas para comercialização direta ao consumidor; e b) O Sistema Brasileiro de Avaliação da Conformidade Orgânica, que é o mecanismo ao qual os agricultores precisam se submeter caso queiram identificar seu produto como orgânico para comercialização via intermediários: supermercados, distribuidores etc.

Consideraç̃̃es sobre a inclusão de pequenos produtores orgânicos brasileiros em mercados de comercialização (BLANC; KLEDAL, 2012) mostram, entretanto, que existem diversos problemas na relação entre esses produtores e a comercialização via intermediários, dentre eles: altos custos para certificação, barreiras estruturais para acesso a crédito, dificuldades na criação de relações de mercado confiáveis e falta de conhecimento sobre gestão organizacional. Segundo esses autores uma parte da assistência técnica e extensão rural - ATER no Brasil, notadamente a executada por organizações ativistas, tem sido eficiente em orientar pequenos produtores para uma transição agroecológica e para a participação em sistemas diretos de venda, 
porém ausente na orientação de processos de gestão para os que estão de alguma forma associados a supermercados, distribuidores e outros atores corporativos.

O impacto das investidas corporativas na produção de orgânicos e a alta conformação dos mercados às estratégias das grandes corporações (THOMPSON; COSKUNER-BALLI, 2007) são realidades que afetam diretamente os pequenos produtores. Esses não possuem recursos para disputar com os grandes e acabam sendo integrados a sistemas produtivos geralmente desvantajosos, cujas relações de poder se estabelecem com um alto grau de dependência dado o custo significativo de recursos de biotecnologia especializada, como as sementes, e também os altos custos de certificação. Tais realidades se mostram mais especificamente nas modalidades de contratos de produção (contract farming) (NAROTZKY, 2016) que se apresentam como a alternativa para os pequenos produtores terem acesso ao suporte tecnológico não oferecido pela ATER.

A problemática das deficiências da ATER brasileira, no que tange aos pequenos produtores de orgânicos, por sua vez, pode estar associada uma dicotomia de suas bases que não dá conta da complexidade dos processos atuais de produção orgânica. Segundo Caporal e Costabeber (2000), tratam-se de dois modelos ligados a noções de sustentabilidade que não conversam entre si. Um primeiro modelo vinculado a uma tecnocracia mundial, que pode ser dito próximo da noção de sistemas agroindustriais, e que afirma "a necessidade de um crescimento econômico continuado, e tenta equacionar a relação sociedade-ambiente e os limites do crescimento mediante um otimismo tecnológico, artifícios econômicos e mecanismos de mercado" (CAPORAL; COSTABEBER, 2000, p. 3); e um segundo modelo que agrupa abordagens críticas da cultura ocidental, ciência e tecnologia modernas com enfoques da economia política que sugerem estratégias alternativas à organização, formas de produção e comercialização, defendendo o respeito a distintos modos de vida e preservação da biodiversidade. "Em síntese, [esse segundo modelo] propõe um câmbio no sistema dominante, em favor de estratégias descentralizadas, compatíveis com as condições ecológicas e capazes de incorporar as identidades étnicas e valores culturais" (CAPORAL; COSTABEBER, 2000, p. 4), estando a agroecologia alinhada com esse segundo modelo.

As agriculturas orgânicas, porém, podem se basear em diferentes práticas de agriculturas (DAROLT, 2010) e possuem, conforme investigação de Blanc e Kledal (2012), sérios problemas mercadológicos que a agroecologia, com seu enfoque local e de mercados diretos, não dá conta. Dessa forma, se pode ver nitidamente um viés tecnopolítico (HECHT, 2001) da agroecologia na PNAPO.

Em termos de tecnologia, entretanto, a questão das escolhas do que se deve utilizar em cada realidade não é um processo apenas técnico e objetivo. Nesse caso, compreensões de como as tecnologias se tornam dominantes e se apresentam como a alternativa aparentemente mais adequada podem produzir boas reflexões e investigações sobre potencialidades, limitações e alternativas às bases tecnológicas de produção e gestão em andamento, colaborando assim para inovação tecnológica organizacional.

\section{TECNOLOGIA COMO REALIZAÇÃO}

As tecnologias se constituem em meio a disputas entre as intenções do design e as subversões do uso, porque são como textos (HINE, 2000) que podem possuir diferentes interpretações a depender dos leitores, que a princípio são livres para entender os usos das tecnologias de formas distintas daquelas que foram intencionadas pelos designers. As tecnologias não são independentes dos sistemas culturais dos quais se originam e nos quais são postas em uso (KLINE, 2003), mas se inserem em um sistema histórico que depende das relações estruturadas entre as pessoas (HARAWAY, 2000).

Há fontes renovadas de poder com as tecnologias, mas essas fornecem ao mesmo tempo fontes renovadas de análise e de ação política (HARAWAY, 2000). Os grupos sempre podem negociar com as formas específicas de poder, autoridade e representação presentes nas tecnologias (ESCOBAR et al., 1994), pois enquanto artefatos culturais, essas são produzidas com objetivos e prioridades situadas em contextos específicos ao mesmo tempo que moldadas pelas formas como são comercializadas, utilizadas, ensinadas e socializadas (HINE, 2000). 
Dessa forma, a abordagem dos Estudos de Ciência, Tecnologia e Sociedade, conhecida com Construção Social da Tecnologia - SCOT propõe o estudo da tecnologia como uma realização, algo que é construído em vez de algo com propriedades intrínsecas, se constituindo assim como uma heurística de pesquisa (BIJKER, 2009) para estudar as mudanças tecnológicas na sociedade em seu contexto histórico e contemporâneo e uma teoria sobre o desenvolvimento da tecnologia e suas relações com a sociedade.

$\mathrm{Na}$ abordagem da construção social o processo de desenvolvimento de uma tecnologia é descrito como uma alternância entre variação e seleção, de forma multidirecional (PINCH; BIJKER, 1987). Se consideradas as variações do desenvolvimento de um artefato, em um determinado período, é possível estudar os porquês das escolhas ou das exclusões de determinadas especificidades da tecnologia, até o ponto em que o artefato adquire certa estabilização. Trata-se de um processo que se inicia devido à flexibilidade interpretativa dos grupos interessados na tecnologia, ou seja, se inicia com a apreensão dos problemas e conjuntos de soluções apresentadas na trajetória de uma tecnologia por variados grupos.

O projeto de uma tecnologia sempre é um processo aberto que pode resultar em diferentes produtos, pois são as circunstâncias do desenvolvimento que definirão em determinado momento a tecnologia enquanto um artefato ou sistema tecnológico dominante, amplamente reconhecido como "a tecnologia" (PINCH; BIJKER, 1987). Partindo-se da identificação dos grupos relevantes associados à tecnologia pode-se verificar como a flexibilidade interpretativa diminui e alguns artefatos alcançam dominância sobre outros. Posteriormente os sentidos convergem, resultando no final em um artefato fruto do processo de construção social (BIJKER, 2009).

Porém, ciência e tecnologia podem ser vistas como "dramas" nos quais a identidade dos atores é sempre problemática (CALLON, 1984). Nesse sentido, avança para além da SCOT uma antropologia simétrica (LATOUR, 1994), que problematiza as identidades pré-estabelecidas de supostos elementos que embasam as escolhas tecnológicas. De fato, na origem das noções da SCOT há parte das bases da antropologia simétrica, notadamente no entendimento que nas controvérsias tecnológicas ou científicas sobre os diferentes objetos se estabelece uma disputa entre os interessados pela caracterização e definição da natureza e funções dos novos objetos científicos ou tecnológicos. Isso é o que Latour (2000) vai denominar posteriormente de laboratórios e contralaboratórios. Ou seja, para toda produção de assertivas sobre um novo objeto há disputas entre diferentes grupos pelo estabelecimento de suas versões do fato.

Entre a SCOT e uma antropologia simétrica há ainda uma compreensão próxima entre os conceitos de estrutura tecnológica e de redes sociotécnicas. Segundo Bijker (1987), estrutura tecnológica é um conceito relacional equivalente às redes sociotécnicas em Callon (1984), que se refere à composição dos diferentes elementos da Ciência e Tecnologia a partir das relações continuadas entre elementos heterogêneos (LATOUR, 2005).

Uma estrutura tecnológica, ou seja, uma mistura de conceitos, técnicas, teorias vigentes, conhecimento tácito, práticas de engenharia e conhecimentos especializados que uma comunidade utiliza para solução de seus problemas (BIJKER, 1987), seria o que providencia os objetivos, ideias e ferramentas para a ação dos atores envolvidos com a tecnologia (BIJKER, 1995), constituindo-se como uma gramática que é utilizada nas interações de um grupo específico interessado na tecnologia, resultando nas atribuições de um sentido compartilhado. Por um lado a estrutura tecnológica indica como o ambiente estrutura o design de um artefato e por outro lado indica como as tecnologias existentes estruturam o ambiente.

Uma compreensão das estruturas tecnológicas passa por diferentes momentos. Primeiro, segundo Bijker (1987), há aquela situação em que a estrutura tecnológica de nenhum dos grupos relevantes é dominante, nessa fase um dos processos que se observa é o alistamento (CALLON, 1984), situação em que outros atores são cooptados a cooperar no processo de estabilização da tecnologia. Posteriormente, a dominância de um dos grupos e sua respectiva estrutura tecnológica emergem. Em um terceiro momento, surgem as disputas por diferentes grupos com estruturas tecnológicas claramente definidas. Por isso um aprofundamento na compreensão dos grupos (ou, no caso da perspectiva de uma antropologia simétrica, das agrupações, porque sempre em movimento e provisórias) é importante.

Pinch e Bijker (1987) apontam para a necessidade de se compreender como os atores definem na prática se um problema é técnico ou social, destacando a fragilidade da fronteira entre esses supostos dois campos. A mesma coisa pode ser dita com relação às fronteiras entre o econômico e o técnico ou o político e o 
técnico (CALLON, 1984). Tais definições, tais indicações, fazem parte do que se pode chamar de retórica da tecnologia (PINCH; ASHMORE; MULKAY, 1992), narrativas fundamentais para se compreender a imbricação de expectativas, crenças, ações e textos associados à tecnologia.

\section{A AGROECOLOGIA: ESTRUTURA TECNOLÓGICA}

As orientações da regulamentação dos orgânicos no Brasil são resultado de um processo de mais de uma década de ações e negociações (MEDAETS; FONSECA, 2005), que define a produção de base agroecológica como um modelo de transição adequado para as atividades interessadas em associar-se à produção orgânica, notadamente com foco em pequenas produções de acordo com uma noção de agricultura familiar.

O que se entende por agroecologia, todavia, vai de um conjunto de abordagens que perpassa movimentos sociais de "reação e de contestação ao domínio técnico industrial e crítico à agricultura de insumos químicos" (BRANDENBURG, 2002, p. 12), passando por um campo do conhecimento para dar conta de práticas de agriculturas alternativas (ASSIS; ROMEIRO, 2002) até a proposição de uma ciência que estabelece um novo modelo de agricultura, baseado no pensamento sistêmico e na noção de agroecossistemas (sistema complexo) por oposição a um modelo convencional de ciência, acusado de apoiar-se nas premissas cartesianas e dominar as práticas de agricultura convencional (sistema simplificado) (ALTIERI, 2004; CAPORAL, 2008).

Para os propositores da agroecologia deve-se considerar as tradições e os conhecimentos locais, assim como direcionar as atenções para "agricultores com poucos recursos, isto é, aqueles que têm o menor acesso aos insumos tecnológicos e poucas relações com o mercado. [Porque a] agroecologia vê esses agricultores como o ponto de partida para uma estratégia de desenvolvimento rural sustentável" (ALTIERI, 2004, p. 42).

No cenário da PNAPO, a agroecologia é o conjunto de práticas indicado para aqueles que se interessam em migrar para a produção orgânica, notadamente aqueles produtores considerados pequenos ou com poucos recursos, a partir do que preconiza a transição agroecológica definida na PNAPO enquanto um "processo gradual de mudança de práticas e de manejo de agroecossistemas, tradicionais ou convencionais, [...] que levem a sistemas de agricultura que incorporem princípios e tecnologias de base ecológica" (BRASIL, 2012). Constituise assim, a agroecologia, como a tecnologia de produção e gestão recomendada para as pequenas agriculturas orgânicas no Brasil.

É possível ver um campo compartilhado de interpretações da agroecologia enquanto tecnologia de gestão adequada para a produção orgânica de pequenos produtores no Brasil a influenciar as políticas públicas. Nesse sentido, se diz, por exemplo, que "a articulação das organizações em rede [da Agroecologia] tenciona o poder público local" (DAMBROS; CAPORAL; PADILLA, 2015, p. 95) e que as caraterísticas do movimento agroecológico, como a autonomia e a cooperação "residem no campo da interdependência mútua compartilhada em rede e ampliada à sociedade geral, salientando a não reprodução das atuais estruturas, mas sim coproduzindo com a natureza suas formas próprias de atuação" (OLIVEIRA, 2016, p. 80), destacando-se que a partir dos anos 1990 houve uma "incorporação do conceito de agroecologia pelas redes da sociedade civil no Brasil" (SCHMITT et al., 2016). Assim, se apresenta uma tecnologia de gestão como mais adequada nas produções das agriculturas ecológicas ou sustentáveis, enfatizando-se que "a organização ou grupo de agricultores para participar do circuito deve fazer parte da rede" (DAROLT et al., 2016, p. 12).

A noção não é nova, mas oriunda das propostas de que um aumento do número de organizações rurais e camponesas, projetos de pesquisa, desenvolvimento e extensão orientados pela agroecologia, vem "gerando uma colaboração em forma de redes para o intercâmbio de experiências e conhecimentos, assim como para fortalecer o consenso [...] buscando incidir [tais propostas] nas políticas de produção rural e desenvolvimento sustentável" (LEFF, 2002, p. 50). Evidencia-se dessa maneira uma percepção de mudança de foco na compreensão e gestão dos processos, nesse caso orientados para que os protagonistas em suas áreas de atuação deixem de "olhar para um leque restrito de inovações [...] [e passem] a se orientar [...] [por um] quadro de hipóteses para a solução dos problemas coletivamente definidos nas redes locais de agricultoresexperimentadores" (PETERSEN; SILVEIRA, 2007, p. 110). 
Dessa forma, a ideia que a agroecologia "se processa mediante a instituição de redes horizontais de interação social" (PETERSEN; SILVEIRA, 2007, p. 104) e até na lógica de que "diversas redes se entrecruzam num processo de reflexividade, avanços e recuos" (ALVES; GUIVANT; GUIVANT, 2010, p. 13) de "organizações e movimentos ligados a agricultores familiares" (ROVER, 2011, p. 57), perfaz o que se pode chamar de um imaginário cultural da agroecologia como constituída a partir de novos processos organizacionais, tecnologia de gestão, para uma agricultura ecológica ou sustentável, com a noção de que iniciativas inovadoras expressamse pela emergência de certa articulação de redes na constituição das políticas públicas nessa seara (CAPORAL; PETERSEN, 2011). Trata-se assim de performances nas quais um dos aspectos observáveis é a tecnologia da agroecologia reconhecidamente ganhando bastante visibilidade e ocupando "espaços importantes em programas e ações de desenvolvimento rural, bem como, no mundo da pesquisa científica, ensino e no movimento social" (ABREU et al., 2011, p. 1).

Todavia, as compreensões derivadas da proposta da agroecologia no Brasil associam os agricultores com poucos recursos tradicionalmente à categoria da agricultura familiar, o que pode ser uma simplificação de diferentes realidades a um processo que possui suas próprias complexidades, perpassando inclusive por especificações que não se aplicam especificamente a agricultores com poucos recursos (GUANZIROLI; BUAINAIN; DI SABBATO, 2012). Além disso, a informação que costuma circular em diferentes meios dando conta de que a produção orgânica está contida em sua maioria na agricultura familiar brasileira (INSTITUTO DE PROMOÇÃO DO DESENVOLVIMENTO, 2010), não considera o estabelecido com a regulamentação da lei dos orgânicos (BRASIL, 2007). Nesse caso, muitos confundem ainda o que pode ser chamado de produção orgânica com produções que estariam mais próximas de uma noção de agricultura ecológica ou sustentável. E nesse sentido, no Brasil, é o termo agroecologia que passa se destacar nas diferentes concepções associadas a essas produções.

\section{${ }_{5}$ CONCLUSÃO}

No presente ensaio apresentou-se uma compreensão em torno da pequena produção orgânica no Brasil, problematizando a agroecologia como estrutura tecnológica dominante nas definições das práticas de produção e gestão adequadas para o contexto dos pequenos produtores.

Argumentando a partir de uma concepção teórica construcionista afirma-se que definições tecnológicas vão além do aspecto puramente técnico e objetivo de seu estabelecimento, constituindo-se como processos sociotécnicos ou tecnopolíticos. Sendo assim, a dominância tecnopolítica da agroecologia privilegia certas abordagens, como a comercialização direta em circuitos locais, não atendendo a pequenos produtores que queiram ou necessitem lidar com circuitos indiretos de comercialização.

Dessa forma, vê-se que se faz necessária pesquisa quanto a outras possibilidades de tecnologias de produção e gestão nessas realidades e a justificativa maior para isso é que há controvérsias sobre a eficácia dos métodos atuais de tecnologia de produção e gestão indicadas para as pequenas agriculturas orgânicas. Métodos que, assim como a dicotomia das abordagens de ATER quanto a sustentabilidade, geralmente estão baseados em uma ponta de noções incompatíveis. De um lado os sistemas a visão tecnocrática e próxima das práticas de contract farming e de outro a visão de crítica à cultura ocidental e com a qual coaduna a tecnopolítica da agroecologia, sendo que essa última se configura como dominante nas disputas pelo que deve ser a tecnologia de produção e gestão dos pequenos produtores de orgânicos.

Um olhar para um grupo específico de produtores, notadamente aqueles que enquanto pequenos precisam ou desejam participar de circuitos de comercialização indiretos junto a atores corporativos, precisaria então pensar diferentes problemáticas da produção orgânica contemporânea no Brasil, como, por exemplo, as nuances do contract farming com seus prós e contras.

As influências na Política Pública das supostas redes de ativismo, ainda que talvez tenham sido importantes para o estabelecimento de uma perspectiva produtiva inclusiva, ou seja, que colocaram a agroecologia no centro das práticas nesse campo, precisam ser vistas também como mecanismos retóricos, porque, olhando para a tecnologia enquanto uma realização é possível compreender que o que a define são 
estruturas de significados que geralmente não dão conta da diversidade dos processos para os quais supostamente aquelas tecnologias seriam as mais adequadas.

\section{REFERÊNCIAS}

ABREU, L. S. de et al. Agroecologia, movimento social, ciência, práticas e políticas públicas: uma abordagem comparativa. Cadernos de Agroecologia, v. 6, n. 2, p. 1-5, 2011. Disponivel em: <http://orgprints.org/22671/>. Acesso em: 6 ago. 2017.

ALTIERI, M. Agroecologia: a dinâmica produtiva da agricultura sustentável. 4. ed. Porto Alegre: Ed. UFRGS, 2004 .

ALVES, A. F.; GUIVANT, J. S.; GUIVANT, J. S. Redes e interconexões: desafios para a construção da agricultura sustentável. Revista Internacional Interdisciplinar INTERthesis, Florianópolis, v. 7, n. 1, p. 1-27, 28 jul. 2010. Disponível em: <http://www.periodicos.ufsc.br/index.php/interthesis/article/view/14245>. Acesso em: 6 ago. 2017.

ASSIS, R. L. de; ROMEIRO, A. R. Agroecologia e agricultura orgânica: controvérsias e tendências. Desenvolvimento e Meio Ambiente, Curitiba, n. 6, p. 67-80, dez. 2002. Disponível em: <http://revistas.ufpr.br/made/article/view/22129>. Acesso em: 17 jun. 2017.

BIJKER, W. E. Of bicycles, bakelites, and bulbs: towards a theory of sociotechnical change. Cambridge: The MIT Press, 1995.

BIJKER, W. E. Social construction of technology. In: OLSEN, J. K. B.; PEDERSEN, S. A.; HENDRICKS, V. F. (Org.). A companion to the philosophy of technology. Chichester: Wiley-Blackwel, 2009. p. 88-94.

BIJKER, W. E. The social construction of Bakelite: toward a theory of invention. In: BIJKER, W. E.; HUGUES, T. P.; PINCH, T. F. (Org.). The social construction of technological systems: new directions in the sociology and history of technology. Massachusets: The MIT press, 1987. p. 159-187.

BLANC, J.; KLEDAL, P. R. The brazilian organic food sector: prospects and constraints of facilitating the inclusion of smallholders. Journal of Rural Studies, v. 28, n. 1, p. 142-154, 2012. Disponível em: <http://dx.doi.org/10.1016/j.jrurstud.2011.10.005>. Acesso em: 15 ago. 2015.

BRANDENBURG, A. Movimento agroecológico: trajetória, contradições e perspectivas. Desenvolvimento e Meio Ambiente, n. 6, p. 11-28. 17 dez. 2002. Disponível em: <http://revistas.ufpr.br/made/article/view/22125>. Acesso em: 17 jun. 2012.

BRASIL. Decreto no 6323, de 27 de dezembro de 2007. Regulamenta a Lei no 10.831, de 23 de dezembro de 2003, que dispõe sobre a agricultura orgânica, e dá outras providências. Disponível em:

<http://www.planalto.gov.br/ccivil_03/_ato2007-2010/2007/Decreto/D6323.htm>. Acesso em: 17 jun. 2015.

BRASIL. Decreto no 7794, de 20 de agosto de 2012. Institui a Política Nacional de Agroecologia e Produção Orgânica. Disponível em: <http://www.planalto.gov.br/ccivil_03/_ato2011-2014/2012/decreto/d7794.htm>. Acesso em: 17 jun. 2015.

BRASIL. Lei no 10.831, de 23 de dezembro de 2003. Dispõe sobre a agricultura orgânica e dá outras providências. Disponivel em: <https://www.planalto.gov.br/ccivil_03/Leis/2003/L10.831.htm>. Acesso em: 20 dez. 2012.

CALLON, M. Some elements of a sociology of translation: domestication of the scallops and the fishermen of St Brieuc Bay. The Sociological Review, v. 32, n. S1, p. 196-233, 1984. 
CAPORAL, F. R. Agroecologia: uma nova ciência para apoiar a transição a agriculturas mais sustentáveise. In: FARIAS NETO, A. L. de (Org.). Savanas: desafios e estratégias para o equilíbrio entre sociedade, agronegócio e recursos naturais. Planaltina: Embrapa Cerrado, 2008. p. 895-929.

CAPORAL, F. R.; COSTABEBER, J. A. Agroecologia e sustentabilidade: base conceptual para uma nova extensão rural. In: WORLD CONGRESS OF RURAL SOCIOLOGY, 10., 2000, Rio de Janeiro. Anais do X World Congress of Rural Sociology. Rio de Janeiro: IRSA/SOBER, 2000. p. 114-123. Disponível em: <http://xa.yimg.com/kq/groups/17929366/420279972/name/PalestraCaporalCostabeber +Agroecologia+e+sustentabilidade.pdf>. Acesso em: 6 ago. 2017.

CAPORAL, F. R.; PETERSEN, P. Agroecologia e políticas públicas na América Latina: o caso do Brasil. Revista de Investigación en Agroecología, v. 6, p. 63-74, 2011. Disponível em:

<http://revistas.um.es/agroecologia/article/view/160681>. Acesso em: 6 ago. 2017.

DAMBROS, O.; CAPORAL, F. R.; PADILLA, M. C. A nova ATER e a formação de redes locais de entidades da agricultura familiar para desenvolver extensão rural agroecológica no Estado do Paraná, Brasil.

EXTRAMUROS - Revista de Extensão da Univasf, v. 3, n. 3, p. 82-96, 2015. Disponível em:

<http://www.periodicos.univasf.edu.br/index.php/extramuros/article/view/589>. Acesso em: 6 ago. 2017.

DAROLT, M. R. As principais correntes do movimento orgânico e suas particularidades. 2010. Atualização online do texto original In: DAROLT, M.R. Agricultura Orgânica: inventando o futuro. Londrina: IAPAR, 2002. Disponível em: <http://docslide.com.br/documents/5-darolt-escolas-da-agricultura-organica.html>. Acesso em: 15 maio 2012.

DAROLT, M. R. et al. Redes alimentares alternativas e novas relações produção-consumo na França e no Brasil. Ambiente \& Sociedade, v. 19, n. 2, p. 1-22, jun. 2016. Disponível em: <http://www.scielo.br/scielo.php?script=sci_arttext\&pid=S1414-753X2016000200002\&lng=en\&tlng=en>. Acesso em: 6 ago. 2017.

ESCOBAR, A. et al. Wenner-Gren Foundation for Anthropological Research Welcome to Cyberia: notes on the Anthropology of Cyberculture [ and Comments and Reply ]. Current Anthopology, v. 35, n. 3, p. 211-231, 1994 .

GUANZIROLI, C. E.; BUAINAIN, A. M.; DI SABBATO, A. Dez anos de evolução da agricultura familiar no Brasil: (1996 e 2006). Revista de Economia e Sociologia Rural, v. 50, n. 2, p. 351-370, jun. 2012. Disponível em: <http://www.scielo.br/scielo.php?script=sci_arttext\&pid=S0103-2003201200020000g\&lng=pt\&tlng=pt>. Acesso em: 23 jun. 2017.

HARAWAY, D. Manifesto ciborgue: ciência, tecnologia e feminismo-socialista no final do século XX. In: SILVA, T, T. (Org.). Antropologia do ciborgue. Belo Horizonte: Autêntica, 2000. p. 33-115.

HINE, C. Virtual ethnography. London: Sage, 2000.

INSTITUTO DE PROMOÇÃO DO DESENVOLVIMENTO - IPD. Perfil do mercado orgânico brasileiro como processo de inclusão social. 2010. Disponível em:

<http://ipd.org.br/upload/tiny_mce/arquivos/Perfil_do_mercado_organico_brasileiro_como_processo_de_incl usao_social.pdf>. Acesso em: 12 jun. 2015.

KLINE, S. J. What is technology. In: SCHARFF, R. C.; DUSEK, V. (Org.). Philosophy of Technology: the technological condition: an anthology. London: Blackwell, 2003. p. 210-212.

LATOUR, B. Ciência em ação: como seguir cientistas e engenheiros sociedade afora. São Paulo: UNESP, 2000.

LATOUR, B. Jamais fomos modernos: ensaio de antropologia simétrica. São Paulo: Ed. 34, 1994.

LATOUR, B. Reassembling the social: an introduction to actor-network-theory. New York: Oxford University Press, 2005. 
LATOUR, B.; HERMANT, E. Paris: invisible city. [2010]. Disponível em: <http://www.brunolatour.fr/virtual/EN/index.html>. Acesso em: 15 maio 2015.

LEFF, H. Agroecologia e saber ambiental. Agroecologia e Desenvolvimento Rural Sustentável, v. 3, n. 1, p. 36-51, 2002. Disponível em: <http://www.pvnocampo.com.br/agroecologia/agroecologia_e_saber_ambiental.pdf>. Acesso em: 6 ago. 2017.

MEDAETS, J. P.; FONSECA, M. F. A. C. Produção orgânica: regulamentação nacional e internacional. Brasília: Ministério do Desenvolvimento Agrário - NEAD, 2005. Disponível em: $<$ http://aao.org.br/aao/pdfs/publicacoes/producao-organica-regulamentacao-nacional-internacional-nead.pdf $>$. Acesso em: 22 jun. 2015.

NAROTZKY, S. Where have all the peasants gone? Annual Review of Anthropology, v. 45, n. 1, p. 301-318, 2016. Disponível em: <http://www.annualreviews.org/doi/10.1146/annurev-anthro-102215-100240>. Acesso em: 25 abr. 2017

OLIVEIRA, N. M. C. de. Agroecologia e recampesinização no contexto da crise agroambiental: abordagens territoriais. Revista Rural \& Urbano, v. 1, n. 1, p. 75-82, 2016. Disponível em: <file://C:/Users/o2/AppData/Local/Temp/10-70-1-PB.pdf>. Acesso em: 6 ago. 2017.

PETERSEN, P.; SILVEIRA, L. Construção do conhecimento agroecológico em redes de agricultoresexperimentadores: a experiência de assessoria ao Pólo Sindical da Borborema. In: PETERSEN, P. (Org.). Construção do conhecimento agroecológico: novos papéis, novas identidades. Rio de Janeiro: Articulação Nacional de Agroecologia, 2007. p. 103-130. Disponível em:

<http://iieb.org.br/files/4813/5215/3881/public_out_construcao_conhecimento.pdf.pdf\#page=105>. Acesso em: 6 ago. 2017.

PINCH, T.; ASHMORE, M.; MULKAY, M. Technology, testing, text: clinical budgeting. In: BJIKER, W. E.; LAW, J. (Org.). Building technology / Shaping society: studies in sociotechnical change. Cambridge: The MIT Press, 1992. p. $265-289$.

PINCH, T. F.; BIJKER, W. E. The social construction of facts and artifacts: or how the sociology of science and sociology of technology might benefit each other. In: BIJKER, W.; HUGHES, T. P.; PINCH, T. (Org.). The social construction of technological systems: new directions in the sociology and history of technology.

Masachusets: The MIT Press, 1987. p. 17-50.

ROVER, O. Agroecologia, mercado e inovação social: o caso da Rede Ecovida de Agroecologia. Ciências Sociais Unisinos, v. 47, n. 1, p. 56-63, 24 maio 2011. Disponível em: <http://revistas.unisinos.br/index.php/ciencias_sociais/article/view/1044>. Acesso em: 6 ago. 2017.

SCHMITT, C. et al. A experiência brasileira de construção de políticas públicas em favor da agroecologia. Brasília: Red PP-AL, 2016. Disponível em: <http://agritrop.cirad.fr/583502/>. Acesso em: 6 ago. 2017.

THOMPSON, C. J.; COSKUNER-BALLI, G. Countervailing Market Responses to Corporate Co-optation and the Ideological Recruitment of Consumption Communities. Journal of Consumer Research, v. 34, n. 2, p. 135-152, 2007. 